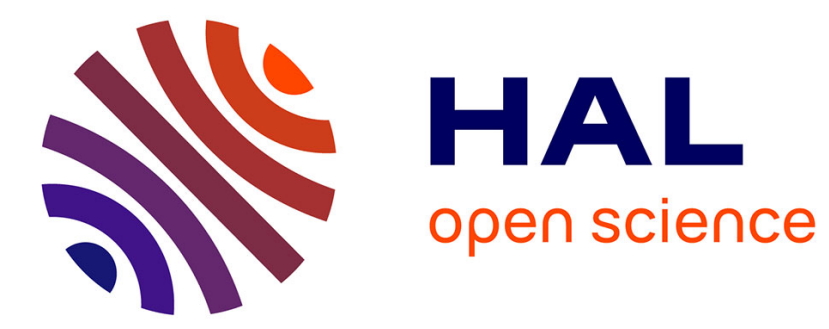

\title{
La loi Faure, une rupture avortée? Effets de policy feedback et application de la loi Faure à Lyon Jérôme Aust
}

\section{To cite this version:}

Jérôme Aust. La loi Faure, une rupture avortée? Effets de policy feedback et application de la loi Faure à Lyon. Politiques et Management public, 2005, 23 (1), pp.53-69. 10.3406/pomap.2005.2260 . hal-01020679

\section{HAL Id: hal-01020679 \\ https://hal-sciencespo.archives-ouvertes.fr/hal-01020679}

Submitted on 8 Jul 2014

HAL is a multi-disciplinary open access archive for the deposit and dissemination of scientific research documents, whether they are published or not. The documents may come from teaching and research institutions in France or abroad, or from public or private research centers.
L'archive ouverte pluridisciplinaire HAL, est destinée au dépôt et à la diffusion de documents scientifiques de niveau recherche, publiés ou non, émanant des établissements d'enseignement et de recherche français ou étrangers, des laboratoires publics ou privés.

\section{(ㅇ)(1) $\$$}

Distributed under a Creative Commons Attribution - NonCommercial - NoDerivatives| 4.0 


\title{
La loi Faure, une rupture avortée ? Effets de policy feedback et application de la loi Faure à Lyon
}

In: Politiques et management public, vol. 23 n 1, 2005. pp. 53-69.

\begin{abstract}
Résumé
En adoptant une grille d'analyse empruntée au néo-institutionnalisme historique, l'article tente de montrer que l'application de la loi Faure à Lyon est structurée par des logiques héritées du système institutionnel antérieur. Si la loi cherche à rompre avec le système des facultés en instituant des universités pluridisciplinaires, elle laisse une place décisive aux acteurs locaux dans la définition des nouvelles universités. Ce faisant, elle fournit un cadre propice à l'expression des logiques antérieures. La définition d'établissements émancipés de la logique facultaire se heurte à deux obstacles majeurs. D'une part, l'héritage institutionnel a contribué à différencier chaque ordre de faculté ; il structure également le choix des instances de concertation. D'autre part, le maintien des découpages antérieurs est l'objet de rendements croissants (increasing returns). Les universitaires lyonnais, engagés sur le sentier facultaire, ont plus intérêt à leur maintien qu'à la promotion de logiques innovantes.
\end{abstract}

Citer ce document / Cite this document :

Aust Jérôme. La loi Faure, une rupture avortée ? Effets de policy feedback et application de la loi Faure à Lyon. In: Politiques et management public, vol. $23 n^{\circ} 1,2005$. pp. 53-69.

doi : $10.3406 /$ pomap.2005.2260

http://www.persee.fr/web/revues/home/prescript/article/pomap_0758-1726_2005_num_23_1_2260 


\title{
LA LOI FAURE, UNE RUPTURE AVORTEE ? EFFETS DE POLICY FEEDBACK ET APPLICATION DE LA LOI FAURE A LYON
}

Jérôme AUST*

Résumé

\begin{abstract}
En adoptant une grille d'analyse empruntée au néo-institutionnalisme historique, l'article tente de montrer que l'application de la loi Faure à Lyon est structurée par des logiques héritées du système institutionnel antérieur. Si la loi cherche à rompre avec le système des facultés en instituant des universités pluridisciplinaires, elle laisse une place décisive aux acteurs locaux dans la définition des nouvelles universités. Ce faisant, elle foumit un cadre propice à l'expression des logiques antérieures. La définition d'établissements émancipés de la logique facultaire se heurte à deux obstacles majeurs. D'une part, l'héritage institutionnel a contribué à différencier chaque ordre de faculté ; il structure également le choix des instances de concertation. D'autre part, le maintien des découpages antérieurs est l'objet de rendements croissants (increasing returns). Les universitaires lyonnais, engagés sur le sentier facultaire, ont plus intérêt à leur maintien qu'à la promotion de logiques innovantes.
\end{abstract}

UMR-CNRS "Triangle" (ENS LSH, IEP de Lyon, Lyon II). Cet article a fait l'objet d'une communication à la journée d'études «Enseignement supérieur et universités : acteurs et institutions " organisée par le RESUP LE 19 septembre 2003 à Dijon. Je tiens ici à remercier l'ensemble des participants à cette journée pour leurs remarques et suggestions. Mes remerciements vont aussi à Christine Musselin et aux membres du groupe "STEP " de l'UMR Triangle pour leurs commentaires sur une première version de ce texte.

Revue POLITIQUES ET MANAGEMENT PUBLIC, Volume 23, n¹, mars 2005.

(C) Institut de Management Public - 2005. 
Introduction

En 1989, soit plus de 20 ans après leur création par la loi Faure, Christine Musselin et Erhard Friedberg décrivent les universités françaises comme des organisations très peu intégrées (Friedberg E. et Musselin C., [16, p. 27). Bien qu'elles se soient récemment affirmées à la faveur de la mise en place de la contractualisation avec le ministère de l'Education nationale (Musselin $C$., [28] et [29] ; Musselin C. et Mignot-Gérard S., [30]) et du développement des relations avec les collectivités locales (Aust J. [2] ; Baraize F. [3]), les universités françaises ont longtemps constitué le parent pauvre du système d'enseignement supérieur français. Le constat opéré par les deux sociologues des organisations à l'orée des années 1990 invite à se replonger dans les conditions de l'application de la loi Faure ${ }^{1}$, instituant les universités après la crise de mai 1968. Sans céder à la tentation d'une quête éperdue des origines, les difficultés de l'affirmation des universités françaises doivent trouver des éléments de compréhension dans leur acte de naissance.

Malgré son caractère cardinal dans l'histoire des universités françaises, la loi Faure n'a pas suscité d'importantes recherches scientifiques. Elle a surtout abouti à la publication d'un nombre impressionnant d'essais prescriptifs sur le sens de la " crise " universitaire française (Bourricaud F., [5] ; Chombart de Lauwe P.H., [9] ; Vuillemin J., [45]) et de témoignages retraçant les conditions difficiles de naissance des universités (Chalendar J. de, [7] ; Debbasch C., [13]). Côté scientifique, les travaux des historiens s'arrêtent souvent avant l'application de la loi Faure (Prost A., [36] ; Girod de l'Ain B., [19]). Quant aux sociologues, ils ont longtemps abordé l'objet université de manière synchronique en se concentrant sur les relations entre tutelle ministérielle, marchés scientifiques et établissements (Friedberg E. et Musselin C., [17] et [18] ; Brisset-Sillon C., [6] ) ou en investiguant sur le fonctionnement concret des universités (Friedberg E. et Musselin C., [16] ; Cohen M.D., March J.G. et Olsen J.P., [11]). Quand les chercheurs incluent la loi Faure dans leur étude (Prost A., [37] ; Musselin C., [29]), la phase d'application de la loi, pourtant décisive, ne retient pas fondamentalement leur attention ${ }^{2}$.

Ce relatif silence scientifique semble cependant devoir être comblé par l'allongement de la période prise en compte pour comprendre l'évolution et le mode de fonctionnement des universités françaises. Dans son dernier ouvrage, Christine Musselin invite ainsi à "adopter une approche plus longitudinale, qui tente d'expliquer d'où venaient et comment se sont maintenus les modes de fonctionnement qui prévalaient auparavant" (Musselin C., [29, p.16]). Partant de la création de l'université impériale, l'auteur montre comment des logiques mises en place au début du XIXème siècle ont continué à marquer, par delà les réformes, la gestion du système d'enseignement supérieur français jusqu'à la fin des années 1980. La centralisation de la gestion administrative, le caractère national des diplômes, l'influence des corporations disciplinaires dans la gestion des carrières restent des caractéristiques structurantes de l'enseignement supérieur français pendant près de deux siècles. C'est en nous intégrant dans cette perspective

1 Loi d'orientation n68-978 sur l'enseignement supérieur du 12 novembre 1968.

2 Le caractère décisif de la période d'application des lois a pourtant été largement souligné par les analyses de l'action publique les plus classiques. Voir par exemple sur ce point Pressman J. L. et Wildavsky A. B., [35] ou Grémion P., [20]. Seule une étude américaine comprend des éléments sur l'application de la loi Faure (Cohen H. S., [12]). 
que nous voudrions envisager l'application de la loi Faure à Lyon. S'il est incontestable que ce texte marque une rupture en instaurant la participation dans les établissements, il nous semble qu'il échoue à créer des établissements réellement pluridisciplinaires. Nous tenterons de montrer que la définition des nouveaux établissements, loin d'être innovante, est dominée par des logiques qui caractérisaient le système antérieur. II s'agit donc ici de comprendre comment les possibilités de changement présentes sont, dans certains cas et à certaines périodes, structurées par les politiques héritées du passé.

Cette question de recherche est l'une des interrogations centrales du néoinstitutionnalisme historique. Ce courant théorique invite à ne plus envisager les institutions comme des variables dépendantes, c'est-à-dire comme le simple résultat des luttes entre des groupes sociaux en concurrence pour l'allocation de ressources publiques rares, mais à les considérer comme des variables indépendantes qui ont un effet propre sur la détermination des résultats sociaux et politiques 1 . Le néo-institutionnalisme historique propose ici un renversement de perspective: les institutions ne sont plus abordées comme des conséquences mais comme des causes. Les néoinstitutionnalistes mettent l'accent sur le fait que les politiques héritées du passé structurent les possibilités de changement présentes ${ }^{2}$. Adopter cette perspective pour comprendre l'application de la loi Faure revient à saisir comment les choix opérés par les prédécesseurs d'Edgar Faure orientent l'application de la réforme par ce que Paul Pierson appelle des " effets de policy feedback ${ }^{3}$. Sans voir dans les institutions la variable expliquant en dernière instance le comportement humain ${ }^{4}$, nous tenterons de montrer dans cet article qu'elles contribuent à structurer l'application de la loi Faure et à orienter le comportement stratégique des acteurs chargés de la mettre en œuvre.

Reste cependant à spécifier les voies de l'influence du passé sur les possibilités de changements présentes. Certains auteurs distinguent l'héritage des politiques publiques passées et les processus de dépendance au sentier institutionnel emprunté (Muller P. et Surel Y., [27, pp. 130-134]). Les études s'appuyant sur la première notion mettent l'accent sur le fait que le legs institutionnel des politiques conduites antérieurement contribue à encadrer les possibilités de changements présentes (Rose R., [38]). La conduite des programmes antérieurs a en effet généré des institutions, résistantes au changement, qui sont autant d'obstacles à la concrétisation d'une rupture avec le passé. Le legs institutionnel contribue donc à enserrer les possibilités de

1 Pour une présentation du programme de recherche du néo-institutionnalisme historique voir Skocpol T. [40] et Thelen K. et Steinmo S., [43].

2 Paul Pierson remarque ainsi qu' " une perspective historique met en lumière le fait qu'aujourd'hui les politiques [policymakers] doivent opérer dans un environnement qui est fondamentalement conditionné par les politiques publiques héritées du passé ". (Pierson P., [33, p. 9]). C'est nous qui traduisons.

3 Les effets de policy feedback sont «les façons dont les choix politiques précédents influencent les processus politiques présents ». Ibid., p.39. C'est nous qui traduisons.

4 Kathleen Thelen et Sven Steinmo notent ainsi que «ce qui est implicite mais crucial dans la plupart des conceptions du néo-institutionnalisme historique est que les institutions contraignent et reflètent les luttes politiques mais qu'elles ne sont jamais les seules causes des résultats produits. " (Thelen K. et Steinmo S., $[43, p .3])$. C'est nous qui traduisons. 
changements présentes dans de strictes limites. D'autres analyses insistent ensuite sur l'influence des institutions passées dans l'orientation de la stratégie des acteurs. S'appuyant sur les travaux des néo-institutionnalistes en économie, les analyses de Paul Pierson notamment mettent l'accent sur le fait qu'une fois les acteurs engagés sur une voie définie, le changement devient plus coûteux que le maintien du choix originel ${ }^{1}$. Le coût d'investissement de départ supporté, il est alors plus rentable de poursuivre toujours dans la même direction plutôt que de tenter de changer de "sentier ». Le maintien du choix premier est ainsi facteur de rendements croissants (increasing returns). C'est au prisme de ces perspectives que nous interrogerons l'application de la loi Faure à Lyon.

L'adoption de cette problématique suppose une stricte définition des institutions prises en compte dans l'analyse. Les néo-institutionnalistes euxmêmes adoptent, en effet, des définitions très variables des institutions (Hall P.A. et Taylor R.C., [21] ; Théret B., [44]). Elles sont associées ici " aux procédures, protocoles, normes et conventions officiels et officieux " (Hall P.A. et Taylor R.C., [21, p. 471]). Nous ne nous limitons pas aux seules structures que la loi cherche à réformer, les facultés. Elles ne sont en effet qu'une composante de ce que Christine Musselin appelle une "configuration universitaire $" 2$. Nous sommes donc conduits à inclure dans l'étude l'organisation institutionnelle des services centraux du ministère de l'Education nationale et celle du Comité Consultatif des Universités (CCU), organe chargé de gérer l'entrée et l'avancement dans la profession universitaire (l'ancêtre du Conseil National des Universités). Enfin, la loi elle-même est une institution: elle oriente donc l'action par les procédures d'application qu'elle définit. En nous appuyant sur un dépouillement archivistique ${ }^{3}$, nous chercherons ici à montrer que la loi Faure, si elle tente bien de promouvoir une rupture avec le système des facultès, favorise paradoxalement, par la place qu'elle accorde aux négociations entre universitaires locaux, l'expression des logiques antérieures (1). Nous tenterons ensuite de démontrer que le legs institutionnel d'un siècle de gestion facultaire constitue un obstacle à la mise en place des universités (2). Le maintien sur le sentier facultaire est, enfin, pour les universitaires lyonnais, facteur de rendements croissants ce qui les pousse à être conservateurs dans l'application de la réforme (3).

1 « Chaque pas dans un sentier (path) particulier a des conséquences qui rendent ce sentier plus attractif pour la prochaine étape ». C'est nous qui traduisons. (Pierson P., [34, p. 253]).

2 Une configuration universitaire désigne " le cadre au sein duquel s'inscrivent, prennent sens et se répondent le type de gouvernement développé par les établissements, le style de pilotage adopté par la tutelle et les modes de régulation interne des disciplines ». (Musselin C., [29, p. 171]).

3 Cet article s'appuie sur le dépouillement des archives rectorales relatives à l'application de la toi Faure à Lyon. (Archives départementales du Rhỏne, série $2690 \mathrm{~W}$, cartons $n^{\circ} 1,2,3,4,5$ et 6). 
La loi Faure, un cadre propice à la résurgence des facultés
Sans présager des intentions d'Edgar Faure et de son entourage ministériel, le contraste entre la radicalité des changements promus dans le texte de loi et la prudence du ministère de l'Education nationale dans la détermination de son dispositif d'application est notable. Le texte de la loi proclame en effet la nécessité de la rupture avec le système ancien des facultés sans cependant que le ministre de l'Education nationale ne s'engage sur la voie de la définition des solutions à apporter. La mise en œuvre de la réforme, pourtant décisive, est largement laissée aux discussions entre les acteurs locaux. Réponse à la crise de mai, la loi Faure illustre la nécessité pour les autorités publiques de rèformer le système d'enseignement supérieur tout autant que les limites de leur pouvoir d'intervention dans le fonctionnement et la définition des nouveaux établissements.

L'ampleur de la crise de mai implique une réforme d'importance. Même s'il s'en inspire, le projet n'est pas le résultat des réflexions conduites sur la nécessité de la réforme de l'enseignement supérieur depuis les années 1950 (Musselin C., [29, p. 57]). Il est au contraire préparé dans l'urgence. Entre la nomination d'Edgar Faure rue de Grenelle et le dépôt du texte à l'Assemblée nationale seulement neuf semaines s'écoulent. Dans ce laps de temps restreint, le ministre prend le temps d'opérer de larges consultations (Chalendar J. de, [7, pp. 20-27]). Le contexte de crise pèse largement sur la tonalité de la loi. Le système facultaire a en effet été radicalement critiqué par les étudiants (Schnapp A. et Vidal-Naquet P., [39, pp. 643-743]). L'autorité des doyens, pierre angulaire du système précédent, a largement été emportée par la crise. Un simple lifting des institutions semble donc politiquement inopportun : la réforme, au moins dans les objectifs qu'elle met en avant, doit être à la mesure de la crise du printemps. La loi rompt donc avec le système ancien des facultés dans trois domaines: les modes de gestion des établissements, leurs relations avec leur autorité de tutelle et leur organisation pédagogique. Les facultés étaient dirigèes par le doyen et par le conseil de faculté (qui réunissait les enseignants de rang $A$ ) assistés d'une assemblée de faculté (qui était ouverte aux autres catégories enseignantes); l'ensemble des acteurs universitaires, enseignants mais aussi étudiants et personnels techniques, participe à la gestion des futures universités. Le législateur dote de plus les nouvelles structures du statut d'établissement public à caractère scientifique et culturel ce qui leur donne une autonomie plus importante sur le plan financier notamment. Enfin, les facultés ètaient organisèes en sections disciplinaires et gérées de façon cloisonnée, les quatre ordres communiquant très peu; les nouvelles universités doivent aux termes de l'article 6 de la loi " associer les arts et les lettres aux sciences et aux techniques".

Les trois piliers de la réforme ont des conséquences importantes sur la définition du dispositif d'application. La création d'établissements pluridisciplinaires implique de mettre à bas les structures passées marquées par le cloisonnement des disciplines et des ordres de faculté. La participation réclame que les acteurs de l'université soient associés à cette refonte institutionnelle. L'autonomie des futurs établissements demande une absence d'autoritarisme du ministère dans la phase d'application. Pour autant, les principes promus dans la loi ne nous semblent pas être les seules causes de la prudence du ministère de l'Education nationale dans la définition des solutions à apporter à la crise de l'enseignement supérieur français. A l'image de la réforme de 1971 sur la fusion des communes [Kervasdoué J. de et 
alii, [22] ; Dupuy F. et Thoenig J.C., [14, pp. 143-154]), la loi Faure et son dispositif d'application permettent de répondre à des impératifs difficilement conciliables: donner des gages d'une volonté réformatrice ambitieuse sans cependant s'engager dans la voie de la prescription des solutions. La conjugaison des principes promus dans la loi et de la situation politique aboutit à un dispositif d'application qui laisse une grande marge de manœuvre aux acteurs locaux. L'application de la loi est marquée par deux temps distincts. Le premier doit aboutir à la définition des unités d'enseignement et de recherche, composantes de base des futures universités. Le second doit s'achever par le regroupement des UER en université. Dans ces deux temps, la marge de manœuvre des acteurs locaux est grande. La création des UER est ainsi entièrement maîtrisée par les universitaires locaux. Les consultations qui doivent amorcer l'application de la loi se veulent extensives et doivent associer l'ensemble des protagonistes de l'université. Le recteur est ainsi chargé d'organiser "les consultations les plus larges possibles de toutes les catégories intéressées (...) y compris les groupes minoritaires et les catégories non représentées " 1 . L'ingérence ministérielle ou rectorale dans la délimitation des UER et dans la rédaction de leurs statuts est proscrite ${ }^{2}$. Le regroupement des UER en universites marque un certain retour des prérogatives du ministère de l'Education nationale dans l'application de la réforme ${ }^{3}$. L'autoritarisme et les capacités d'initiative du ministère de l'Education nationale ne doivent cependant pas être surestimés. Les instances centrales ne cherchent pas à imposer des découpages élaborés dans le secret du cabinet ministériel. Elles tentent plus habilement de générer une discussion sous contrainte.

La marge de mancouvre des universitaires locaux est d'autant plus grande que la loi est extrêmement floue et imprécise sur ce que doit être ou ne doit pas être une UER ou une université 4 . Seules l'impossibilité de la transformation d'une faculté en université et la nécessité de l'application de la pluridisciplinarité sont sans cesse rappelées aux acteurs locaux. La loi ne fait donc que donner des procédures, qu'èlaborer des règles de concertation entre les acteurs sans présumer des orientations qui seront retenues par les protagonistes de la mise en œuvre. Le ministère de l'Education nationale refuse même, alors que les universitaires lyonnais le demandent, d'intervenir dans la rédaction des statuts des unités et des universités en rappelant leur autonomie. La loi Faure semble donc proche de la logique des politiques constitutives. Comme elles, elle «ne dit pas qu'elle est la définition du problème et quelles sont les modalités de son traitement opérationnel. Elle se contente de définir des procédures qui servent de contexte d'action sans que

1 Carton $n^{\circ} 2$, instructions ministérielles envoyées au recteur d'académie, note d'information $n^{\circ} 1$, octobre 1968 , p. 3.

2 Carton $n^{\circ} 2$, instructions ministérielles envoyées au recteur d'académie relatives aux statuts des universités et des UER, 25 novembre 1968, p. 2.

3 Un envoyé du ministère de l'Education nationale précise ainsi aux universitaires lyonnais que « le ministre s'est résenvé le remodelage des universités. II les veut pluridisciplinaires. Certes, si sur le plan local, les unités récemment créées proposent des universités qui soient de dimension raisonnable et pluridisciplinaires, le ministre en tiendra compte ". Carton $n^{\circ} 2$, compte-rendu de la réception de Mr Praderie au rectorat d'académie. 15 octobre 1968.

4 II semble que les premières versions du texte aient été encore plus floues. Le Conseil d'Etat a ainsi cherché à préciser les dispositions législatives lors de son examen du texte de loi. (Chalendar J. de, [7, pp. 26-27]). 
soit présupposé pour autant le degré d'accord et d'implication des acteurs retenus » [Duran P. et Thoenig J.C., [15, p. 601]). L'absence d'information sur la situation qui prévaut à l'intérieur de chaque établissement, la spécificité de chaque contexte local et la proximité des événements de mai poussent le ministre à renvoyer la définition des mesures à prendre aux discussions qui doivent se tenir entre les universitaires.

La loi Faure est donc largement le produit de la crise de mai 1968. Le texte législatif, dans sa lettre, promeut un changement radical qui tente de mettre en place des établissements autonomes, démocratiques et pluridisciplinaires. La déclinaison de ces trois objectifs et les conditions politiques aboutissent cependant à laisser la création des nouveaux établissements aux mains des universitaires locaux. Ce dispositif décentralisé constitue un cadre propice à la résurgence des logiques facultaires au sein des universités. Reste à en identifier les voies. L'héritage institutionnel des politiques déjà mises en œuvre constitue le premier obstacle à la concrétisation de la rupture.

\section{L'héritage institutionnel comme obstacle à la rupture}

Tout d'abord, l'application de la loi Faure est marquée par la prégnance des institutions que des siècles de gestion facultaire ont contribué à mettre en place. Le legs institutionnel détermine la mise en place des structures de concertation et rend difficile les rapprochements qui pourraient aboutir à des regroupements inédits et novateurs, conformes à l'esprit de la loi d'orientation.

Les institutions antérieures pèsent tout d'abord sur la mise en place des instances de concertation nécessaires à l'application de la loi. Dans les deux phases de la réforme, les structures utilisées rééditent les découpages institutionnels antérieurs. Pour discuter de la définition des UER, le recteur d'académie choisit de s'appuyer sur les commissions paritaires mises en place par les grévistes de mai 1968. L'organisation de ces commissions est cependant calquée sur les découpages facultaires. II existe ainsi une commission paritaire par section. Seules ces instances disposent aux yeux des syndicats enseignants de la légitimité nécessaire pour être les pivots de l'application de la réforme ${ }^{1}$. La nécessité du décloisonnement disciplinaire est certes invoquée mais dans des structures qui réaniment les découpages précédents. On discute donc la pluridisciplinarité de section à section sans qu'aucune instance transversale ne soit mise en place ce qui ne favorise pas la formalisation de rapprochements novateurs.

Pendant la période de regroupement des UER en universités, ce sont les universitaires lyonnais, récemment élus dans les conseils d'UER, qui discutent de la composition des futurs établissements. Plusieurs projets pluridisciplinaires sont élaborés par des conseils d'unités. Leur préparation solitaire les condamne cependant à l'échec. Les projets sont souvent envoyés par l'intermédiaire du recteur d'académie aux conseils d'unités concernés qui n'ont pas été associés à la réflexion sur le regroupement possible. L'inexistence de liaison entre les UER rend impossible la formalisation d'un projet collectivement adopté. Indépendantes et isolées, les UER constituent

1 Carton $n^{\circ} 6$, note du 17 septembre 1968 des syndicats SGEN/SNESup Iyonnais au recteur d'Académie. 
donc un ensemble extrêmement éclaté oủ les mécanismes de régulation des intérêts divergents sont difficiles à trouver. Nous avons plus affaire ici à une constellation d'organisations qu'à un ensemble intégré. Les conseils d'UER ne parviennent pas à s'imposer comme les instances de base qui discutent de la mise en œuvre de la réforme. Ce sont les conseils transitoires de gestion (CTG) des facultés qui vont servir de pivots pour les négociations. Structures provisoires destinées à gérer les affaires courantes en attendant la mise en place des universités, ils sont basés sur le découpage facultaire ${ }^{1}$. Là encore, les instances qui servent de base à la concertation entre universitaires lyonnais réaniment les découpages antérieurs. La discussion sur l'application de la loi se fait donc de faculté à faculté ce qui ne favorise pas le dépassement du cadre institutionnel passé.

La gestion cloisonnée des différents ordres de faculté a également contribué à créer des différences marquées dans la gestion des carrières et dans l'organisation des établissements ce qui complexifie les conditions de leur fusion. Facultés "professionnelles" (médecine et droit) et facultès " académiques" héritières de la faculté des arts (lettres et sciences) ont évolué de façon très diverse (Mayeur F., [26]). Médecine et droit disposent d'une agrégation du supérieur qui ouvre la voie au professorat. En sciences et en lettres, un tel concours n'existe pas. Victor Cousin tente sans succès de doter ces deux ordres d'une agrégation sur le modèle de la médecine et du droit en 1840 (Mayeur F., [26, p. 13]). Les disciplines des facultés de sciences et de lettres ne disposent donc que d'une agrégation du secondaire. L'institutionnalisation de la sociologie et de la psychologie à la fin des années 1950 dans les facultés de lettres ajoute à la diversité. Ces nouvelles disciplines ne disposent d'aucune agrégation. L'utilisation des assistants n'est pas non plus la même selon l'ordre de faculté considéré. Ainsi dans les lettres et les sciences, les assistants sont nombreux et essentiels au fonctionnement des établissements. En droit, ils jouent un rôle bien moindre et en médecine ils sont quasiment en voie d'extinction dans les années 1960. Leur statut juridique (fonctionnaire ou non) et leur rémunération sont des plus variables selon les ordres de faculté considèrés. Sur le plan de l'organisation des études, la création des Centres Hospitalo-Universitaires (CHU) en reliant un centre hospitalier à une facultè de médecine ${ }^{2}$ contribue à singulariser les études médicales par rapport aux autres facultès. Cette spécificitè des ètudes médicales conduit les médecins lyonnais à ne pas participer aux discussions avec les autres disciplines pendant la définition des unités d'enseignement et de recherche. Chaque UER médicale est ainsi reliée à un CHU.

Les politiques menées dans le passé ont également structuré l'organisation de l'instance de gestion corporatiste des carrières (le CCU) et du ministère de l'Education nationale. Or, la loi Faure ne remet pas en cause l'organisation institutionnelle de ces deux structures. Le ministère de l'Education nationale, qui gère les diplômes, reste découpé disciplinairement. Le CCU, qui gère les

1 Les conseils transitoires de gestion sont mis en place par le décret $n^{\circ} 68-1104$ du 7 décembre 1968 .

2 Sur l'application de la réforme Debré qui relie certains hôpitaux aux facultés de médecine voir les travaux fondateurs d'Haroun Jamous (Jamous H., [23]) 
carrières enseignantes, n'est pas fondamentalement réorganisé lui non plus 1 . Les universitaires ne sont pas seulement les employés d'une faculté donnée: ils sont aussi voire beaucoup plus les membres d'une discipline. Si les facultés sont supprimées, le maintien de l'influence des disciplines dans la gestion des carrières enseignantes fait pression pour la conservation des découpages disciplinaires au sein des nouvelles unitès d'enseignement et de recherche. II est même remarquable que certaines disciplines (la gestion par exemple) qui ne disposaient pas de section au sein du système facultaire profitent de la loi Faure pour accéder au statut d'UER. Paradoxalement, l'application de la réforme aboutit parfois à un recul de la pluridisciplinarité. Dans les rares unités qui tentent l'expérience de la pluridisciplinarité, la recherche d'une certaine indépendance disciplinaire est notable. Ainsi, les sociologues, engagés dans un projet de fusion avec la psychologie et la philosophie, demandent l'établissement d'un véritable contrat "délimitant le domaine et précisant les conditions d'une relative autonomie de la sociologie par rapport aux deux autres disciplines ${ }^{2}$. En réformant les facultés, la loi Faure ne s'attaque qu'à un seul pilier de la structuration disciplinaire du système d'enseignement supérieur français. Les réformateurs, en ne touchant pas à l'organisation du CCU et du ministère de l'Education nationale, se privent d'un levier de changement majeur ${ }^{3}$.

Au total, l'héritage institutionnel est lourd à porter. Les instances de concertation sont marquées par les découpages précédents. Près de deux siècles de gestion cloisonnée entre les disciplines ont contribué à spécifier chaque ordre de faculté et à asseoir au sein du ministère et du CCU une gestion disciplinaire qui ne facilite pas le dépassement du système facultaire. Ce ne sont cependant pas là les seuls obstacles qui vont se poser à une application innovante de la loi Faure à Lyon. Les universitaires Iyonnais se comportent en effet en héritiers du système précédent : loin de bouleverser le système des facultés, ils tendent à le reproduire à l'intérieur des nouveaux établissements.

1 Le CCU est simplement quelque peu réorganisé par le décret du 10 mai 1969. Le texte ne touche cependant pas au caractère disciplinaire du découpage du CCU. (Chevallier J., [8, p. 19]).

2 Carton $n^{\circ} 3$, projet de protocole d'accord intersections en vue de la constitution d'une unité de psychologie, philosophie et sociologie, document dactylographié, non daté.

3 Erhard Friedberg et Christine Musselin remarquent ainsi que «le changement des universités ne passe pas tant par une transformation de leurs structures organisationnelles intemes que par une action indirecte de structuration/restructuration des systèmes plus larges qui régulent l'organisation des champs disciplinaires et les rapports entre les universités et leurs administrations de tutelle. ». Friedberg E. et Musselin C. [16, p. 188]). 
Les rendements croissants comme obstacles à l'innovation
Les universitaires lyonnais se montrent particulièrement peu innovants dans les propositions qu'ils font au ministère de l'Education nationale. Dans les deux phases de l'application de la loi, la préservation des découpages antérieurs constitue leur stratégie essentielle. Seules trois unités à la faculté des lettres et des sciences humaines, une unité à la faculté de droit et une unité à la faculté des sciences ne sont pas directement issues des sections des anciennes facultés. Pendant la période de définition des universités, les médecins lyonnais cherchent à obtenir coûte que coûte la transformation de leur faculté en université malgré les rappels ministériels de l'impossibilité de cette option. Les scientifiques, s'ils ne sont pas défavorables aux additions d'unités, tentent de maintenir les liens existants entre les composantes de l'ancienne faculté. $\mathrm{Au}$ sein des facultés de lettres et de droit, les universitaires sont moins attachés à la préservation du cadre facultaire. Un projet réunissant les sciences sociales au sein d'un établissement commun est même un temps envisagé. II échoue pourtant sur la question de l'implantation géographique (Aust J., [2, pp.197-211]). Après de longs mois de négociations, aucun projet collectivement adopté ne réussit à voir le jour. Le ministère de l'Education nationale, devant l'enlisement des projets, demande aux universitaires lyonnais d'aboutir et de proposer des regroupements. Malgré la pression ministérielle, scientifiques et médecins demandent la transformation de leur faculté en université, solution écartée dès l'origine par le ministère 1 . Les unités issues des facultés de lettres et de droit arrivent à un compromis et demandent la réunion de leurs deux établissements ${ }^{2}$. II ne s'agit cependant pas dans l'esprit des acteurs de l'époque d'une fusion. L'acceptation du projet est en effet soumise à une condition: que les UER issues de la faculté de droit et celles issues de la faculté de lettres disposent du même nombre de sièges au sein de l'assemblée constitutive provisoire de la future université. Le cadre facultaire reste bien le prisme par lequel les acteurs locaux appréhendent la mise en place de la réforme. Les acteurs cherchent, dans les nouvelles universités, à garantir la représentation égalitaire des deux anciennes facultés. Le ministère prend acte de cet accord, crée l'établissement et, devant l'absence de projets concernant les sciences et la médecine, impose la fusion de ces deux anciennes facultés. Le découpage ministériel ne s'affranchit pas du cadre facultaire ; deux universités sont constituées, Lyon I, qui groupe bloc à bloc sciences et médecine, et Lyon II qui réunit, lettres et droit. La création des universités lyonnaises aboutit à la juxtaposition de facultés deux à deux dans des établissements communs. Les universitaires lyonnais se conduisent donc en dignes héritiers du système antérieur. Comment interpréter cette attitude?

Ce comportement peu innovant ne nous apparaît pas être le seul produit d'un refus politique de la réforme Faure et des événements de 1968. Les quatre facultés ont en effet vécu très diversement la crise du printemps: si les facultés de sciences et de lettres ont été très touchées par la contestation, les facultés de médecine et de droit surtout l'ont beaucoup moins été. II est de plus peu probable, au vu de leur simple nombre, que les universitaires lyonnais partagent tous le même avis sur la loi d'orientation. Cette attitude nous semble pouvoir notamment s'expliquer par une convergence des intérêts

1 Carton $n^{\circ} 3$, courrier du recteur d'académie au ministre de l'Education nationale du 4 octobre 1969.

2 Ibid. 
à maintenir les découpages antérieurs dans l'application de la loi. Maintenir le découpage disciplinaire et facultaire au sein des nouvelles universités est moins risqué que jouer le jeu des nouvelles institutions. La conservation de l'organisation antérieure est en effet l'objet de rendements croissants (increasing retums). Engagés sur le sentier facultaire, les universitaires peuvent perdre beaucoup au changement de direction qui est proposé par la loi. L'argument n'explique pas toutes les attitudes: nous l'avons dit d'autres éléments rentrent en compte. La mise en place d'une UER est ainsi directement imputable à des divisions idéologiques ${ }^{1}$. Le projet de création de l'universitè des sciences sociales bute avant tout sur la question de son implantation géographique. Reste qu'au total la dépendance au sentier institutionnel emprunté nous apparaît être un élément qui structure l'orientation stratégique des acteurs de la réforme dans la stricte période d'application de la loi Faure (novembre 1968-décembre 1969). Trois domaines sont sujets aux rendements croissants : les processus daction collective, les processus cognitifs d'interprétation du monde et de légitimation et les processus de développement institutionnel (Pierson P., [34]).

Les processus d'action collective sont d'abord sujets à des rendements croissants. L'efficacité d'une mobilisation dépend avant toute chose du nombre de personnes qu'elle concerne. Une mobilisation sur une base inédite est plus difficile à opérer qu'une mobilisation sur un socle déjà constitué. Jouer le jeu de la loi Faure demande la création d'une coalition favorable à l'éclatement des institutions précédentes et la construction d'un projet commun et collectivement adopté. Cette stratégie implique donc de supporter un investissement de départ important (convaincre les autres de se mobiliser) et comprend la possibilité d'un échec. A l'inverse, une stratégie défensive qui vise à préserver les découpages antérieurs n'implique pas un tel travail de construction. Les institutions passées fournissent un socle commun à la mobilisation. Les promoteurs d'une mise en place effective de la loi doivent donc construire ex nihilo un projet consensuel. Les étudiants qui avaient violemment remis en cause les institutions facultaires ne forment pas une force de proposition dans l'application de la loi. Ne parvenant pas à se coordonner, restant même parfois volontairement en marge du processus d'application ${ }^{2}$, le front étudiant à Lyon est plus un axe du refus de la loi Faure qu'une force de proposition constructive. Si la présence étudiante contribue à politiser les discours, elle ne joue pas un rôle décisif dans la formalisation et la définition des projets pendant la phase d'application de la loi.

Les processus d'interprétation cognitif du monde et de légitimation sont eux aussi soumis à des rendements croissants. L'acceptation de coopérations nouvelles (par exemple entre le droit et l'histoire ou la sociologie au sein d'une UER ou d'une université commune) demande de supporter des coûts

1 La mise en place de l'UER d'études des langues italienne et néo-latines semble directement imputable à des considérations idéologiques. Les prises de position de son directeur contre "la minorité révolutionnaire » (carton $n^{\circ} 3$, lettre au recteur d'académie du 14 décembre 1968) et les réactions étudiantes qu'elles provoquent (carton $n^{\circ} 3$, communiqué étudiant : «Comment les nostalgiques du mandarinat récupèrent les conquêtes de mai », novembre 1968) expliquent la naissance de cette unité.

2 Certains groupes décident d'ailleurs de ne pas « rentrer dans le jeu des institutions qui leur sont proposées par la loi d'orientation ». Carton $n^{\circ} 2$, courrier de l'unité de philosophie au recteur d'académie du 10 décembre 1968. 
d'investissement élevés liés à la diffusion de la nouvelle représentation. Dès lors que la légitimité d'une coopération est établie, les coûts d'investissement ne sont plus à supporter. Sa légitimité lui vient de son antériorité ; elle relève de la quasi-évidence. Les découpages qui tendent à reproduire les divisions facultaires apparaissent ainsi plus légitimes que ceux qui s'en écartent. L'argument de la complémentarité pédagogique et scientifique est souvent mobilisé par les universitaires lyonnais. C'est le cas par exemple de l'ancienne section de français qui, en ne se divisant pas, cherche à ne pas " compromettre les rapports étroits qui doivent exister entre les enseignements de littérature française, d'études médiévales, de littérature comparée et de langues françaises " 1 . L'une des rares UER pluridisciplinaire qui se met en place réunie la sociologie, la psychologie et l'ethnologie. Ces trois disciplines cohabitaient déjà au sein de la même section à la faculté des lettres. Leur complémentarité scientifique et pédagogique apparaît ainsi déjà légitime. La légitimité antérieure des coopérations scientifiques marque également le processus de définition des universités. Un projet propose ainsi de séparer histoire de l'art, histoire et philosophie qui collaboraient au sein de la faculté des lettres. Les historiens de l'art le refusent au motif des complémentarités qui unissent ces disciplines et des liens étroits qu'elles doivent conserver au sein des futures universités 2 .

Les processus de développement institutionnel sont enfin soumis à des mécanismes de rendements croissants. Les institutions précédentes ont contribué à forger des habitudes de gestion et de coopération entre universitaires lyonnais. Dès lors que leur fonctionnement est jugé satisfaisant, les acteurs de la mise en place de la réforme sont incités à les maintenir. Casser les liens existants entre les unités issues d'une même faculté ou accepter la fusion avec d'autres disciplines dans une UER commune, c'est prendre le risque d'être marginalisé dans la gestion des nouvelles institutions. Les institutions passées réalisent ici un équilibre de Nash : les acteurs locaux adhèrent à ces modèles parce qu'ils ont plus à perdre qu'à gagner dans leur remise en cause. Il est ainsi remarquable que la plupart des attitudes innovatrices soit le fait de disciplines marginalisées dans l'organisation facultaire. Pendant la période de définition des universités, ce sont les économistes, les sociologues et les psychologues qui semblent les plus prompts à dépasser les découpages facultaires antérieurs. Ces universitaires sont ceux qui bénéficient des positions les moins établies au sein du système précédent. Ils ont ainsi beaucoup moins à perdre que les autres dans la promotion de regroupements émancipés des découpages facultaires. A l'inverse, leurs collègues dès lors qu'ils sont satisfaits du fonctionnement des facultés ont beaucoup plus à perdre qu'à gagner dans une application innovante de la loi. Ce n'est pas un hasard si ce sont les médecins et les scientifiques qui défendent le plus vigoureusement leurs anciennes facultés. Ce sont en effet eux qui disposent des crédits de recherche et de

\footnotetext{
1 Carton $n^{\circ} 3$, courrier de l'assemblée paritaire de français au Ministre de l'Education nationale du 14 novembre 1968.

2 Carton $n^{\circ} 3$, courrier du directeur de l'UER d'histoire de l'art au recteur d'académie du 21 juin 1969.
} 
fonctionnement les plus importants ${ }^{1}$. Les pouvoirs des futurs conseils d'université dans le domaine de la répartition des crédits font craindre la coopération avec des UER issues de facultès différentes ${ }^{2}$.

\section{Conclusion}

En décembre 1969 sur le plan de la mise en place des structures, le changement qui résulte de l'application de la loi Faure à Lyon semble loin de la lettre du texte législatif. Très peu d'UER sont pluridisciplinaires et leur découpage est, dans l'extrême majorité des cas, calqué sur celui des sections. Les deux universités créées se résument à la juxtaposition bloc à bloc des anciennes facultés. La situation lyonnaise, si elle est emblématique des effets de policy feedback, n'est pas une exception. Dans les villes universitaires de province comptant un nombre d'étudiants qui interdit la constitution d'une seule universitè (Aix-Marseille, Bordeaux, Grenoble, Lille, Montpellier, Nancy, Rennes, Strasbourg, Toulouse), l'application de la loi aboutit à des résultats comparables. Si certaines universitès s'émancipent des découpages précédents et associent des unités qui sont issues de facultés différentes (Lille 1 associe ainsi des économistes, des géographes, des psychologues, des sociologues et des scientifiques soient des unités issues de trois établissements distincts), les découpages facultaires constituent le plus souvent la matrice des nouvelles universités. Si les combinaisons varient très largement, les unités issues des facultés de sciences et de médecine sont les plus enclines à maintenir les découpages antérieurs: à Montpellier, à Bordeaux, à Toulouse, à Nancy les unités de médecine et de sciences conservent les liens qu'elles entretenaient dans l'ancien système. Seuls certains médecins d'Aix-Marseille, certains mathématiciens de Lille et les membres de l'IREPS de Strasbourg font un choix distinct de celui de leur faculté d'origine. Les unités issues des facultés de droit et de lettres semblent, comme à Lyon, les plus enclines à s'émanciper du découpage facultaire. Pour autant, les anciens liens entre les unités sont préservés à Nancy. Toulouse, Montpellier. Pour le reste, ce sont essentiellement les économistes (à Lille, à Strasbourg, à Montpellier) les géographes (à Strasbourg, à Lille, à AixMarseille), les psychologues et les sociologues (à Aix-Marseille, à Lille, à Bordeaux) qui sont les plus favorables à des regroupements innovants (Association d'étude pour l'expansion de l'enseignement supérieur, [1, pp. 209-359]). Si seule une analyse approfondie des contextes locaux permet de saisir les logiques des regroupements qui s'opèrent, la fréquence de la préservation des découpages précédents nous semble bien signaler au moins l'importance du système facultaire dans l'application de la loi Faure. Comparée à la lettre de la loi d'orientation et à sa promotion ambitieuse de la pluridisciplinarité, la concrétisation du changement est bien timide. Pour autant, l'échec de la loi d'orientation n'est pas si sûr.

1 Les différences dans les dotations en crédits de recherche sont mème explicitement mentionnées par les scientifiques comme étant un frein à l'ètablissement de liens qui s'affranchissent du cadre facultaire. Carton $n^{\circ} 3$, compte-rendu de la réunion des CTG des 4 facultés lyonnaises et du recteur d'académie du 23 mai 1969.

2 Rétrospectivement, cette crainte apparaît infondée. En effet, les conseils d'université ne parviendront jamais à s'affirmer comme des instances d'arbitrage sur le plan budgétaire. (Musselin C.. [29, pp. 64-65]). Pour autant, au moment de l'application de la loi Faure, ces inquiétudes peuvent apparaître fondées. 
Même si les modes de fonctionnement des universités restent longtemps marqués par les logiques facultaires (CNE, [10, p. 138]), la réforme Faure réussit à redonner un cadre institutionnel viable à la majeure partie des établissements universitaires après une crise extrêmement grave. La loi d'orientation permet, sans prendre le risque politique de prescrire des solutions, une « remise en institution » des établissements d'enseignement supérieur. Cette pacification n'aboutit cependant pas dans tous les cas. La situation lyonnaise est, là encore, emblématique. A l'instar de son homologue aixoise, l'université Lyon II, groupant les lettres et le droit, rentre dès la réunion de son assemblée constitutive dans une crise qui aboutit à la scission de l'établissement et à la constitution de deux établissements concurrents, Lyon II et Lyon III. Ici, ce ne sont ni l'appartenance aux deux anciennes facultés ni les allégeances disciplinaires qui structurent le choix entre les deux universités mais bien des clivages très composites qui comprennent notamment des rivalités personnelles et des divisions idéologiques issues de mai 1968 (Bayard F. et Comte B. [4, pp. 77-98]). Les effets de " policy feedback " ne sont donc plus ici déterminants. Ces crises notées, la loi, et c'est son premier succès, permet de redonner des institutions à l'enseignement supérieur français. Elle ouvre également la gestion des universités aux étudiants et aux personnels non enseignants. A plus long terme surtout, la loi Faure permet des réformes ultérieures. En s'appuyant sur les établissements mis en place à la fin des années 1960, le ministère de l'Education nationale parvient, à travers les contrats quadriennaux, à renforcer le niveau universitaire (Musselin C., [28] et [29] ; Musselin C. et Mignot-Gérard S. [30]). A l'image d'autres réformes conduites dans le domaine de la protection sociale (CSG, RMI...), la réforme Faure est assimilable à une "réforme innovatrice " (Palier $B$. et Bonoli G., [31, p. 412]) qui permet de s'écarter timidement du sentier initial tout en proposant des opportunités de changement aux réformateurs postérieurs. Si les crises peuvent conduire à des réorientations profondes de la gestion de certains domaines d'action publique (Surel $Y$. [41] et [42]), l'analyse de l'application de la loi Faure atteste de l'absence de relation mécanique entre une crise et un changement majeur à court terme. Elle montre également que le changement par la loi est un processus bien contingent dont les effets dépassent souvent l'horizon temporel des réformateurs qui le promeuvent. II reste le bricolage incertain que décrivait déjà à sa manière Charles $E$. Lindblom (Lindblom C.E. [24] et [25]). 


\section{BIBLIOGRAPHIE}

[1] Association d'étude pour l'expansion de l'enseignement supérieur (1971), De l'université aux universités. Octobre 1968-janvier 1971, Paris, Armand Colin, 1971, $815 \mathrm{p}$.

[2] AUST J. (2004), Permanences et mutations dans la conduite de l'action publique. Le cas des politiques d'implantation universitaire dans l'agglomération lyonnaise (1958-2004). Thèse de doctorat de science politique sous la direction de Gilles Pollet, Université Lyon II, 2004, $337 p$

[3] BARAIZE F. (1996), " La notion de secteur est-elle encore centrale ? La négociation du sens dans l'enseignement supérieur ", Pôle sud, $n^{\circ} 4$, mai 1996, pp. 107-122.

[4] BAYARD F. et COMTE B. (2004), L'université Lyon 2. 1973-2004, Lyon, PUL, 2004, $378 \mathrm{p}$.

[5] BOURRICAUD F. (1971), Universités à la dérive. France, Etats-Unis, Amérique du Sud, Paris, Stock, 1971, $178 \mathrm{p}$.

[6] BRISSET-SILLION C. (1997), Universités publiques aux Etats-Unis. Une autonomie sous tutelle, Paris, L'Harmattan, 1997, 299 p.

[7] CHALENDAR J. de (1970), Une loi pour l'université, Paris, Desclée de Brower, 1970, $269 \mathrm{p}$.

[8] CHEVALLIER J. (1971), L'enseignement supérieur, Paris, PUF, 1971, $96 \mathrm{p}$.

[9] CHOMBART DE LAUWE P.H. (1968), Pour I'Université. Avant, pendant et après mai 1968, Paris, Payot, 1968, $173 \mathrm{p}$.

[10] CNE (1993): Universités : la recherche des équilibres. Rapport au Président de la République. 1989-1993, Paris, La Documentation française, 1993, $153 \mathrm{p}$.

[11] COHEN M.D., MARCH J.G. et OLSEN J.P. (1972), " A Garbage Can Model of Organizational Choice ", Administrative Science Quaterly, vol. $17, n^{\circ} 1$, mars 1972, pp. 1-25.

[12] COHEN H.S. (1978), Elusive Reform : The French Universities. 19681978, Boulder, Westview Press, 1978.

[13] DEBBASCH C. (1971), L'université désorientée. Autopsie d'une mutation, Paris, PUF, 1971, 173 p.

[14] DUPUY F. et THOENIG J.C. (1983), Sociologie de l'administration française, Paris, Armand Colin, 1983, $206 \mathrm{p}$.

[15] DURAN P. et THOENIG J.C. (1996), « L'Etat et la gestion publique territoriale ", Revue française de science politique, vol. $46, n^{\circ} 4$, août 1996, pp. 580-622.

[16] FRIEDBERG E. et MUSSELIN C. (1989), En quête d'universités. Etudes comparées des universités en France et en RFA, Paris, L'Harmattan, 1989, $220 \mathrm{p}$.

[17] FRIEDBERG E. et MUSSELIN C. (dir.) (1992), Le gouvernement des universités. Perspectives comparatives, Paris, L'Harmattan, 1992, 351 $p$.

[18] FRIEDBERG E. et MUSSELIN C. (1993), L'Etat face aux universités en France et en Allemagne, Paris, Economica, 1993, $186 \mathrm{p}$.

[19] GIROD DE L'AIN B. (1989), "La corporation universitaire et l'Etat ", Sociologie du travail, vol. $31, n^{\circ} 4,1989, p p .477-491$. 
[20] GREMION P. (1976), Le pouvoir périphérique. Bureaucrates et notables dans le système politico-admnistratif français, Paris, Le Seuil, 1976, $477 \mathrm{p}$.

[21] HALL P.A. et TAYLOR R.C. (1997), " La science politique et les trois néoinstitutionnalismes ", Revue française de science politique, vol. 47, n’34, juin-août 1997, pp. 469-495.

[22] KERVASDOUE J. de, FABIUS L., MAZODIER M. et DOUBLET F. (1976), «La loi et le changement social : un diagnostic. La loi du 16 juillet 1971 sur les fusions et regroupements de communes », Revue française de sociologie, $n^{\circ} 3$, juillet 1976, pp. 423-450.

[23] JAMOUS H. (1969), Sociologie de la décision. La réforme des études médicales et des structures hospitalières, Paris, Editions du CNRS, 1969,257 p.

[24] LINDBLOM C.E. (1959), "The Science of Muddling Through », Public Administration Review, vol. 19, $n^{\circ} 2$, printemps 1959, pp. 79-88.

[25] LINDBLOM C.E., (1979), "Still Muddling, not yet Through », Public Administration Review, vol. $39, n^{\circ} 6$, novembre-décembre 1979, pp. 517 526.

[26] MAYEUR F. (1985), " L'évolution des corps universitaires (1877-1968) » dans Charle $C$. et Ferré $R$. (dir.), Le personnel de l'enseignement supérieur en France auX XIXé et XXe siècles, Paris, Editions du CNRS, 1985, pp. 11-26.

[27] MULLER P. et SUREL Y. (1998), L'analyse des politiques publiques, Paris, Montchrestien, 1998, $156 \mathrm{p}$.

[28] MUSSELIN C. (1997), " Etat, Université, la fin du modèle centralisé ? ", Esprit, $n^{\circ} 234$, juillet 1997, pp.18-29.

[29] MUSSELIN C. (2001), La longue marche des universités françaises, Paris, PUF, 2001, $218 p$.

[30] MUSSELIN C. et MIGNOT-GERARD S. (2003), « L'autonomie, pas à pas », Sciences de la société, $n^{\circ} 58$, février 2003 , pp. 17-35.

[31] PALIER B. et BONOLI G. (1999), "Phénomènes de path dependence et réformes des systèmes de protection sociale ", Revue française de science politique, vol. 49, $\mathrm{n}^{\circ} 3$, juin 1999, pp. 399-419.

[32] PIERSON P. (1993), "When effect becomes Cause. Policy Feedback and Political Change ", World Politics, vol. 45, n4, 1993, pp. 595-628.

[33] PIERSON P. (1994), Dismantling the Welfare State? Reagan, Thatcher and the Politics of Retrenchment, Cambridge, Cambridge University Press, 1994, 213 p.

[34] PIERSON P. (2000), "Increasing Returns, Path Dependence and the Study of Politics », American Political Science Review, vol. 94, n², 2000, pp. 251-267.

[35] PRESSMAN J.L. et WILDAVSKY A.B. (1974), Implementation, Berkeley, California University Press, 1974, 182 p.

[36] PROST A. (1968), L'enseignement en France 1800-1967, Paris, Armand Colin, 1968, $524 \mathrm{p}$.

[37] PROST A. (1992), Education, socièté et politiques. Une histoire de l'enseignement supérieur en France de 1945 à nos jours, Paris, Seuil, 1992, 226 p.

[38] ROSE R. (1990), "Inheritance before Choice in Public Policy », Journal of Theroretical Politics, vol. 2, n³, 1990, pp. 263-291. 
[39] SCHNAPP A. et VIDAL-NAQUET P. (1988 - $1^{\text {ère }}$ édition 1969), Journal de la commune étudiante. Textes et documents. Novembre 1967-juin 1968, Paris, Seuil, 1988, $860 \mathrm{p}$.

[40] SKOCPOL T. (1985), « Bringing the State Back In : Strategies of Analyses in Current Research " dans Evans P. B., Rueschmeyer D. et Skocpol T. (dir.), Bringing the State Back In, New York, Cambridge University Press, 1985, pp. 107-163.

[41] SUREL Y. (1997), L'Etat et le livre. Les politiques publiques du livre en France (1957-1993), Paris, L'Harmattan, 1997, 362 p.

[42] SUREL Y. (1997), " Quand la politique change les politiques. La loi Lang du 10 août 1981 et les politiques du livre ", Revue française de science politique, vol. 47, $n^{\circ} 2$, avril 1997, pp. 147-172.

[43] THELEN K. et STEINMO S. (1992), " Historical Institutionalism in comparative Politics " dans Steinmo S., Thelen K. et Longstreth F. (dir.), Structuring Politics. Historical Institutionalism in Comparative Analysis, Cambridge, Cambridge University Press, 1992, pp. 1-32.

[44] THERET B. (2000), «Institutions et institutionnalismes. Vers une convergence des conceptions de l'institution ? " dans Tallard M., Théret B. et Uri D. (dir.), Innovations institutionnelles et territoires, Paris, L'Harmattan, 2000, pp. 25-68.

[45] VUILLEMIN J. (1968), Rebâtir I'Université, Paris, Fayard, 1968, 82 p. 